\title{
Thrombocytopenic purpura with depot medroxyprogesterone acetate and subsequent use of contraceptive implant: making decisions in the absence of evidence
}

\author{
Ruth Holman, Gillian Stephen
}

\section{Case report}

A 19-year-old woman presented to her general practitioner with a purpuric rash 3 weeks after receiving her first $150 \mathrm{mg}$ intramuscular injection of depot medroxyprogesterone acetate (DMPA) at a family planning clinic. She had no medical or family history of note and was taking no other medication. Following investigation by the haematology department she was diagnosed with thrombocytopenic purpura. Her platelet count at diagnosis was $4 \times 10^{9} / 1$ (local laboratory normal range: $\left.100-150 \times 10^{9} / 1\right)$. Other haematological and biochemical indices were normal. Three weeks prior to the administration of DMPA the patient had a full blood count taken before a minor surgical procedure under general anaesthesia, and on that occasion her platelet count was normal.

Two months after receiving DMPA the patient's platelet count remained low (in the range $2-4 \times 10^{9} / 1$ ). She returned to the family planning clinic to discuss her contraceptive options. Since DMPA may have been a causal factor in the development of her thrombocytopenia there was concern that other progestogens might have a similar effect. However, other progestogens are reversible: DMPA is not.

Despite an extensive literature search, no evidence was identified to support an association between other hormonal contraceptives and thrombocytopenic purpura. No information was identified to guide clinicians and patients on appropriate contraceptive use with thrombocytopenic purpura. This patient had used combined oral contraception in the past but expressed concern that she might not take it reliably, and this had been a factor in choosing DMPA. She did not wish to use an intrauterine device or system.

After counselling the patient opted for a subdermal progestogen-only implant (Implanon ${ }^{\circledR}$ ). Following discussion about the potential risks, and with the agreement of her haematologist, the implant was inserted. This was 14 weeks after the first injection of DMPA. The patient was not at risk of pregnancy at this time. Her platelet count at insertion was $8 \times 10^{9} / 1$. Her platelet count was assessed weekly. The implant was to be removed if her platelet count fell. The insertion procedure was uneventful. She was advised to contact the family planning clinic if she developed swelling or excessive tenderness at the site of insertion. Her platelet count returned to normal 5 months after her first

J Fam Plann Reprod Health Care 2005; 31(4): 333-334

(Accepted 1 February 2005)

Department of Sexual Health, Ayrshire Central Hospital, Irvine, UK

Ruth Holman, MRCOG, MSc, Consultant in Sexual and Reproductive Health

Department of Obstetrics and Gynaecology, University of Aberdeen, Aberdeen, UK

Gillian Stephen, BSc, Research Assistant

Correspondence to: Dr Ruth Holman, Department of Sexua Health, Ayrshire Central Hospital, Kilwinning Road, Irvine KA12 8SS, UK. E-mail: ruth.holman@aapct.scot.nhs.uk
DMPA injection while she continued with the progestogenonly implant. The patient was reviewed 3 months after the implant was inserted and she was happy to continue with this method. She had had no bleeding since insertion.

The Medicines and Health Care products Regulatory Agency were informed via the Committee on Safety of Medicines 'Yellow Card' system.

\section{Discussion}

Thrombocytopenia is a blood disorder characterised by low platelets. ${ }^{1}$ It has acute and chronic forms ${ }^{1}$ and is most commonly idiopathic. ${ }^{2}$ Bleeding is uncommon unless the platelet count falls below $10-20 \times 10^{9} / 1 .^{3}$ Acute idiopathic thrombocytopenia (ITP) occurs particularly in children. It usually improves spontaneously and, in children at least, rarely recurs. Chronic ITP is more common in adults, and occurs in three times as many women as men. ${ }^{1}$ Chronic ITP is characterised by persistent thrombocytopenia that is usually secondary to an $\mathrm{IgG}$ antibody against glycoprotein complexes Ib/IX and/or IIb/IIIa. The clinical features include skin petechiae, large bruises and mucosal bleeding causing nose bleeds, gingival bleeding or melaena. ${ }^{1}$ Menorrhagia is a common feature: a study on the clinical and haematological profiles of ITP found that $50 \%$ of the female patients had menorrhagia. ${ }^{4}$ Severe bleeding such as intracranial or intra-abdominal bleeding is rare. 1

Virtually every medication has been associated with thrombocytopenia and the platelet destruction is usually IgG-mediated. ${ }^{3}$ Patients with drug-induced thrombocytopenia typically have moderate to severe thrombocytopenia seen 1-2 weeks after commencing medication. ${ }^{3}$ The thrombocytopenia usually resolves quickly after stopping the drug. ${ }^{3}$ Patients should not take the drug again as it will cause thrombocytopenia with subsequent exposure. ${ }^{3}$

When the patient presented we were concerned about the risk of recurrence of thrombocytopenia with other contraceptive methods. Given the timing of events it seemed likely that DMPA was the causal factor in her thrombocytopenia, although other factors such as her recent general anaesthetic could not be entirely discounted. The association with DMPA seems more likely in retrospect as it is apparent that the platelet count began to climb at about the time the injection would be expected to be wearing off. A small study in three women following $150 \mathrm{mg}$ DMPA administered via intramuscular injection observed serum medroxyprogesterone following a single injection. ${ }^{5}$ Levels of medroxyprogesterone acetate (MPA) gradually declined and remained relatively constant at $1 \mathrm{ng} / \mathrm{ml}$ for 2-3 months following a single injection, declining thereafter to $0.2 \mathrm{ng} / \mathrm{ml}$ by the sixth month. Levels were undetectable at 7-9 months. In some women MPA can be identified in serum for as long as 9 months after injection. ${ }^{5,6}$ It was not known if there would be any interaction with other progestogens thus prolonging the thrombocytopenia or making it worse.

A literature search of MEDLINE and EMBASE for the period 1996-2004 found little evidence to guide clinical practice. Physician prescribing information for DMPA for 
doctors in the USA identified that in a large trial of almost 4000 women, blood dyscrasia was an adverse reaction following DMPA in $<1 \%$ of subjects. ${ }^{7}$ Fraser et al. reported on three cases of thrombotic thrombocytopenic purpura in users of the levonorgestrel implant (Norplant ${ }^{\circledR}$ ). ${ }^{8}$ Due to the small number of cases these researchers identified, they could not establish a causal relationship between thrombotic thrombocytopenic purpura and the levonorgestrel implant.

MPA is a 17-OH progesterone-derived pregnane, and etonorgestrel is a testosterone-derived gonane. ${ }^{9}$ No evidence was found to indicate whether this difference would be significant in predicting whether etonorgestrel would cause thrombocytopenia if MPA had.

On the basis of clinical experience we surmised that as thrombocytopenic purpura is associated with menorrhagia, the IUD might be a less suitable option. The levonorgestrel-releasing intrauterine system (LNG-IUS), which is licensed for use in the management of idiopathic menorrhagia, might be a suitable alternative. There was no evidence to identify whether systemic absorption of the intrauterine progestogen would affect the platelet count. Insertion of a subdermal implant may lead to haematoma formation in women with low platelets. No contraindication to oral contraception was found other than the theoretical risk of reaction to the progestogen component. MPA is not used in any other contraceptives but is present in some forms of hormone replacement therapy (HRT), and the patient might be wise to avoid such formulations should she require HRT in the future.

Although clinicians endeavour to practice evidencebased medicine, there is often a lack of evidence to guide practice and decisions have to be made on the basis of theory and expert opinion. Whilst it can be discomfiting and difficult to communicate such uncertainty to patients, it is important that they understand the basis on which decisions are being made.

This case report suggests that it is possible to use an alternative progestogen after a suspected drug-induced thrombocytopenia to an injectable progestogen.

\section{Acknowledgement}

The patient gave written consent to this case report being published.

Statements on funding and competing interests

Funding. None identified.

Competing interests. None identified.

References

1 Reilly JT, Cawley JC. Haematological disease. In: Axford J (ed.), Medicine. Oxford, UK: Blackwell Science, 1996.

2 Moake JL. Thrombotic thrombocytopenic purpura today. Hosp Pract (Off Ed) 1999; 34: 53-59.

3 Webert KE, Kelton JG. Disorders of platelet number and function. In: Warrell D, Cox TM, Firth JD, Benz EJ (eds), Oxford Textbook of Medicine. Oxford, UK: Oxford University Press, 2004.

4 Khan M, Rahid MA, Rahman J. Clinical and haematological profile of idiopathic thrombocytopenic purpura. Journal of Medical Teachers' Federation 2000; 5: 16-19.

5 Ortiz A, Hirol M, Stanczyk FZ, Goebelsmann U, Mishell DR. Serum medroxyprogesterone acetate (MPA) concentrations and ovarian function following intramuscular injection of depo-MPA. J Clin Endocrinol Metab 1977; 44: 32-38.

6 Mishell DRJ, Kharma KM, Thorneycroft IH, Nakamura RM. Oestrogenic activity in women receiving an injectable progestogen for contraception. Am J Obstet Gynecol 1972; 113: 372-376.

7 Pharmacia. Depo-Provera. 2003. http://www.medicines.org.

8 Fraser JL, Millenson M, Malynn ER, Uhl L, Kruskall MS. Possible association between the Norplant contraceptive system and thrombotic thrombocytopenic purpura. Obstet Gynecol 1996; 87: $860-863$.

9 Druckmann R. Classification and pharmacology of progestogens. Gynaecol Forum 2004; 9: 5-10.

\section{vasectomy services}

\section{Over 100,000 men have chosen us...}

making Marie Stopes International the country's most experienced vasectomy provider. With 20 centres nationwide we are able to offer a quick, reliable and straightforward service with no waiting lists.

For men who want the convenience of counselling, assessment and procedure on one day we offer a same day service. Our "non scalpel vasectomy technique" takes just a few minutes with no stitches and we provide all post operative aftercare and semen testing.

If you or your patient would like more information, simply call the number below and we will send further details.

\section{Call us on 08451203644 - 24 hours}

\section{or visit www.mariestopes.org.uk}

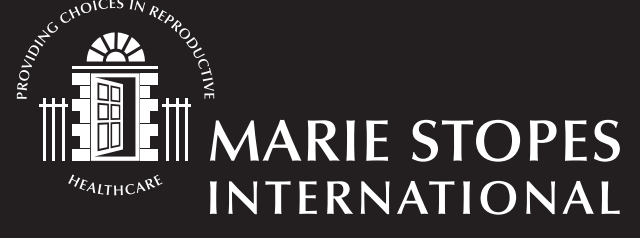

